\title{
Dynamics of C-phycocyanin in various deuterated trehalose/water environments measured by quasielastic and elastic neutron scattering
}

\author{
Ingo Köper · Sophie Combet $\cdot$ Winfried Petry $\cdot$ \\ Marie-Claire Bellissent-Funel
}

Received: 9 August 2007/Revised: 28 November 2007/ Accepted: 6 December 2007/Published online: 8 January 2008 (C) EBSA 2007

\begin{abstract}
The molecular understanding of protein stabilization by the disaccharide trehalose in extreme temperature or hydration conditions is still debated. In the present study, we investigated the role of trehalose on the dynamics of the protein C-phycocyanin (C-PC) by neutron scattering. To single out the motions of C-PC hydrogen $(\mathrm{H})$ atoms in various trehalose/water environments, measurements were performed in deuterated trehalose and heavy water $\left(\mathrm{D}_{2} \mathrm{O}\right)$. We report that trehalose decreases the internal C-PC dynamics, as shown by a reduced diffusion coefficient of protein $\mathrm{H}$ atoms. By fitting the Elastic Incoherent Structure Factor-which gives access to the "geometry" of the internal proton motions-with the model of diffusion inside a sphere, we found that the presence of trehalose induces a significantly higher proportion of immobile C-PC hydrogens. We investigated, by elastic neutron scattering, the mean square displacements (MSDs) of deuterated trehalose/ $\mathrm{D}_{2} \mathrm{O}$-embedded C-PC as a function of temperature in the range of 40-318 K. Between 40 and $\sim 225 \mathrm{~K}$, harmonic MSDs of C-PC are slightly smaller in samples containing trehalose. Above a transition temperature of $\sim 225 \mathrm{~K}$, we observed anharmonic motions in all trehalose/water-coated C-PC samples. In the hydrated
\end{abstract}

I. Köper · S. Combet $(\bowtie) \cdot$ M.-C. Bellissent-Funel Laboratoire Léon-Brillouin UMR 12 CEA/CNRS, CEA/Saclay, 91191 Gif-sur-Yvette Cedex, France e-mail: sophie.combet@cea.fr

I. Köper · W. Petry

Fakultät für Physik E13, Technische Universität München, 85747 Garching, Germany

Present Address:

I. Köper

Max Planck Institute for Polymer Research,

55128 Mainz, Germany samples, MSDs are not significantly changed by addition of $15 \%$ trehalose but are slightly reduced by $30 \%$ trehalose. In opposition, no dynamical transition was detected in dry trehalose-embedded C-PC, whose hydrogen motions remain harmonic up to $318 \mathrm{~K}$. These results suggest that a role of trehalose would be to stabilize proteins by inhibiting some fluctuations at the origin of protein unfolding and denaturation.

\section{Introduction}

Trehalose is a non-reducing disaccharide of glucose isolated from algae, bacteria, fungi, insects, invertebrates, and yeasts, as well as from lower vascular plants and a few flowering plants (Clegg and Filosa 1961; Fox 1995). This sugar has been found in large amounts in organisms able to survive extreme external stresses, such as high or very low temperatures or periods of complete drought (Clegg and Filosa 1961; Crowe et al. 1996; Fox 1995). In presence of trehalose, the structure of the biomaterial is conserved and life and decay processes are suspended or highly slowed down (Clegg and Filosa 1961; Fox 1995). These astonishing bioprotecting properties of trehalose have been confirmed by in vitro experiments in lipids, proteins, viruses, and mammalian cells (Bieganski et al. 1998; Carpenter and Crowe 1989; Crowe et al. 1984; Eroglu et al. 2000; Sola-Penna et al. 1998). Furthermore, trehalose has been found to be the most effective bioprotectant in cryopreservation and dessication (Bieganski et al. 1998; Green and Angell 1989; López-Díez et al. 2004), as well as in thermal preservation (Sola-Penna et al. 1998).

However, the molecular mechanisms of the protective role of trehalose remain poorly understood and are still 
debated (Cordone et al. 2005). The main hypotheses are the following ones:

1. The "high viscosity" hypothesis, according to which the denaturation processes are hindered, is based on the very high glass transition temperature of trehalose solutions (Affouard et al. 2005; Branca et al. 1999; Cornicchi et al. 2005; Giuffrida et al. 2006; Green and Angell 1989; Magazù et al. 2007; Mazzobre et al. 1997; Rector et al. 2001). The amorphous phase formed by trehalose may protect biomolecules at low temperature, since the formation of ice crystals, followed by the destruction of cellular material, is hindered.

2. The "water entrapment" mechanism, according to which trehalose concentrates water close to the biomolecule, as shown by infrared and Raman spectroscopy for the residual water molecules in the dry state (Belton and Gil 1994) and by molecular dynamics simulations for trehalose/water-coated CO-myoglobin (MbCO) (Cottone et al. 2002).

3. The "water replacement" theory, according to which trehalose would replace water molecules normally present at the surface of the biomaterial to conserve its structure. Several observations suggest direct interactions, through hydrogen bonds, between the sugar and the polar groups of proteins and phospholipids (Belton and Gil 1994; Carpenter and Crowe 1989; Cottone et al. 2002; Crowe et al. 1984; Giuffrida et al. 2003; Librizzi et al. 2002). López-Díez et al. (2004) emphasized the relatively higher stability of hydrogen bonds with the protein in the case of trehalose, as compared to other bioprotecting agents. Moreover, Sola-Penna et al. (1998) suggested that more water molecules can be substituted by trehalose than by other sugars, through its higher hydration volume.

Moreover, to stabilize a protein, the bioprotecting sugar may induce, in extreme conditions as well as at ambient temperature, a slowing down of some protein molecular motions that are responsible for unfolding and denaturation. Indeed, myoglobin or haemoglobin $\mathrm{CO}$ rebinding after flash-photolysis showed a strongly inhibited protein dynamics and a decreased escape of $\mathrm{CO}$ from the heme pocket in trehalose matrix (Gottfried et al. 1996; Hagen et al. 1995). Mössbauer and optical absorption spectroscopy described an analogous large reduction of the nonharmonic motions of the heme iron atom in $\mathrm{MbCO}$ embedded in trehalose (Cordone et al. 1998). This is in good agreement with the decreased amplitude of nonharmonic motions reported for $\mathrm{MbCO}$ by molecular dynamics simulations in presence of trehalose (Cottone et al. 2001). Mean square displacements (MSDs), measured by elastic neutron scattering, of hydrogen atoms in trehalose-coated MbCO (Cordone et al. 1999; Librizzi et al. 2001), as well as in glucose-embedded lysozyme (Cornicchi et al. 2006), have shown absence of anharmonic dynamics in dry condition.

Previously, we performed inelastic and quasielastic neutron scattering experiments to study the influence of trehalose on C-phycocyanin (C-PC) dynamics. C-PC is a phycobiliprotein present in the antennae of cyanobacteria and is involved in the first steps of photosynthesis (Glazer 1989). C-PC has been widely used as a model protein to study water motions at its surface by neutron scattering (Bellissent-Funel et al. 1996). By comparing the dynamics of hydrated samples of C-PC and of trehalose-coated C-PC, we observed, in a time range from picoseconds to several nanoseconds, that trehalose induces a slowing down of the motions of the protein up to two orders of magnitude (Köper et al. 2002). By using the model of a particle diffusing inside a sphere (Volino and Dianoux 1980), we showed that trehalose reduces the proportion of mobile hydrogens in the protein and that the confining sphere radius is independent on trehalose content (Köper et al. 2002).

The aim of the present study was to further investigate, by quasielastic neutron scattering, the nature and the geometry of C-PC hydrogen motions in various deuterated trehalose $/ \mathrm{D}_{2} \mathrm{O}$ environments with the high resolution of the backscattering neutron spectrometer IN13 at the Institut Laue-Langevin (ILL, Grenoble, France). Moreover, we report, by elastic neutron scattering, the evolution of C-PC proton MSDs as a function of temperature in the range of 40-318 K. Previous studies have already shown the evolution, as a function of temperature, of the MSDs of trehalose-coated MbCO (Cordone et al. 1999; Librizzi et al. 2001). In these studies, the samples were either fully protonated - and measured only in the dry state-(Cordone et al. 1999) or exchanged with $\mathrm{D}_{2} \mathrm{O}$ before lyophilizationand studied both in the dry and the $\mathrm{D}_{2} \mathrm{O}$-rehydrated states-(Librizzi et al. 2001). In the present study, we used deuterated trehalose and $\mathrm{D}_{2} \mathrm{O}$ to reduce their contributions in incoherent neutron scattering measurements, in order to single out C-PC dynamics, by measuring the motions of the non-exchangeable hydrogen atoms of the protein. Furthermore, we investigated C-PC dynamics in various trehalose/water environments in order to understand up to which extent trehalose could inhibit protein motions.

\section{Materials and methods}

\section{Sample preparation}

Protonated C-PC was prepared by Dr. Henry L. Crespi (Argonne National Laboratory, Argonne, IL, USA) from 
the cyanobacteria Synechococcus lividus, as previously described (Hattori et al. 1965). Trehalose was deuterated according to a method previously detailed (Köper et al. 2005). Briefly, deuterated trehalose was obtained by catalytic exchange reactions using a Raney nickel catalyst. The degree of deuteration of protons in the sugar has been determined by ${ }^{13} \mathrm{C}$-NMR to about $80 \%$ (Köper et al. 2005). The C-PC/trehalose samples were prepared by mixing dry powders of protonated protein and deuterated trehalose at the desired ratio (see Table 1). They were solubilized in heavy water and dialysed to exchange all labile protons against deuterons (three times in $\mathrm{D}_{2} \mathrm{O}$ at $4^{\circ} \mathrm{C}$ ). Finally, samples were frozen in liquid nitrogen and exposed to vacuum to sublimate water. This co-lyophilization ensured a homogeneous distribution of trehalose molecules around the protein. An amount of about $200 \mathrm{mg}$ of powder was held in a standard aluminium cell with internal spacing of $0.5 \mathrm{~mm}$ and then hydrated under heavy water atmosphere. The level of hydration was determined by weighing the samples on a high precision balance. The cell was finally closed by an indium seal before neutron measurements. The C-PC/ $\mathrm{D}_{2} \mathrm{O}$ sample (\#1), previously measured, had been prepared in the same conditions (Dellerue 2000).

\section{Neutron scattering experiments}

Quasielastic and elastic neutron scattering experiments were performed for all the samples (including sample \#1, Dellerue 2000) on IN13 thermal backscattering spectrometer (French-Italian Collaborative Research Group) at the ILL. This spectrometer has the advantage to give access to a large $Q$-range, from 0.28 to $4.75 \AA^{-1}$, together with a very good energy resolution [Full Width at Half Maximum (FWHM) of $\sim 8 \mu \mathrm{eV}$ with an incident wavelength of $2.3 \AA$ ], which gives access to dynamics on a time scale above $150 \mathrm{ps}$ and up to $400 \mathrm{ps}$.

We performed quasielastic neutron scattering measurements on $\mathrm{D}_{2} \mathrm{O}$-hydrated powders of protonated C-PC with various deuterated trehalose contents in order to observe individual motions of the non-exchangeable protons of the protein. The use of hydrated powders allows to avoid contribution from the global protein motions (rotations and translations), as it is the case in protein solutions (Pérez et al. 1999). Due to the low neutron flux on the instrument and the relatively weak quasielastic neutron scattering from the samples, data collection times for quasielastic scans from $-100 \mu \mathrm{eV}$ up to $+100 \mu \mathrm{eV}$ were of about 3 days per sample to obtain sufficient statistics. Quasielastic data were corrected for empty cell contribution and normalized to a vanadium scatterer using standard ILL software.

For the elastic neutron scattering scans, we also used powders of protonated C-PC and deuterated trehalose at different ratios and hydrated at different levels with $\mathrm{D}_{2} \mathrm{O}$. Measurements were performed in the temperature range of $40-318 \mathrm{~K}$ with a step of 5-10 $\mathrm{K}$ between each measurement and a time counting of $90 \mathrm{~min}$ per temperature. For each temperature, the data have been analysed using Eq. 4 (see below) over a $Q$-range up to $Q=4.75 \AA^{-1}$, except at $T=318 \mathrm{~K}$, for which the analysis was only performed up to $Q=4.5 \AA^{-1}$. We have subtracted the empty aluminium cell to all spectra. Samples were assumed to be elastic scatterers at the lowest temperature $(40 \mathrm{~K})$ and, so, spectra at $40 \mathrm{~K}$ were used as a vanadium to normalize those at higher temperatures.

Quasielastic and elastic neutron scattering theory

Thermal neutron scattering is a spectroscopic technique which is well-suited to measure protein motions on an atomic length scale and a pico- to nanosecond timescale (Smith 1991; Zaccai 2000). Since the incoherent neutron scattering cross-section of hydrogen nucleus exceeds, by at least one order of magnitude, the scattering cross-sections (coherent and incoherent) of all other atoms present in biological samples (mainly $\mathrm{C}, \mathrm{O}, \mathrm{N}, \mathrm{P}$, and $\mathrm{S}$ ), as well as of the deuterium atom, total scattering is dominated by incoherent scattering of individual $\mathrm{H}$ atoms. The incoherent scattering from C-PC $\mathrm{H}$ atoms, i.e. from its nonexchangeable $\mathrm{H}$ atoms (69\% of the total C-PC $\mathrm{H}$ atoms), contributes to $87 \%$ of protein total scattering signal. As hydrogen atoms are abundant and uniformly distributed in proteins, the scattering represents a mean global view of

Table 1 Sample composition, percentage of trehalose and $\mathrm{D}_{2} \mathrm{O}$ content, and contribution of sample components as a percentage of the total neutron scattering signal from the sample

\begin{tabular}{|c|c|c|c|c|c|c|}
\hline & \multirow[t]{2}{*}{ Sample composition ( $w: w: w)$} & \multirow{2}{*}{$\begin{array}{l}\text { Trehalose content } \\
(w: w)\end{array}$} & \multirow{2}{*}{$\begin{array}{l}\mathrm{D}_{2} \mathrm{O} \text { content } \\
(w: w)\end{array}$} & \multicolumn{3}{|c|}{ Contribution to total neutron scattering } \\
\hline & & & & $\mathrm{C}-\mathrm{PC}$ & Trehalose & $\mathrm{D}_{2} \mathrm{O}$ \\
\hline$\# 1$ & $\mathrm{C}-\mathrm{PC} / \mathrm{D}_{2} \mathrm{O} \quad 1: 0.5$ & - & $33 \%$ & $91.2 \%$ & - & $8.8 \%$ \\
\hline$\# 2$ & C-PC/trehalose/ $\mathrm{D}_{2} \mathrm{O}$ 1:0.3:0.7 & $15 \%$ & $35 \%$ & $81 \%$ & $8.1 \%$ & $10.9 \%$ \\
\hline \#3 & C-PC/trehalose/ $\mathrm{D}_{2} \mathrm{O}$ 1:0.75:0.75 & $30 \%$ & $30 \%$ & $71.8 \%$ & $17.9 \%$ & $10.3 \%$ \\
\hline \#4 & C-PC/trehalose $1: 1$ & $50 \%$ & - & $75 \%$ & $25 \%$ & - \\
\hline
\end{tabular}


protein dynamics. Besides, as we used deuterated trehalose/water environment, we probed mainly the dynamics of the protonated C-PC protein: C-PC scattering signal represents between 72 and $91 \%$ of the total scattering signal from the samples (see Table 1).

We performed two types of experiments: (1) the quasielastic mode to measure the incoherent dynamical structure factor $S_{\text {inc }}(Q, \omega)$, which provides the probability for an incident neutron to be scattered by the sample with a momentum transfer $\hbar \mathrm{Q}$ and an energy transfer $\hbar \omega$, where $\hbar$ is the reduced Planck constant; (2) the elastic scan mode, without energy transfer consideration, to get Debye-Waller factors and, by analysing the data as a function of the square modulus of the scattering vector $Q^{2}$, to obtain the MSDs $\left\langle u^{2}\right\rangle$ of the scattering particles.

In quasielastic neutron scattering, the incoherent dynamical structure factor $S_{\mathrm{inc}}$ can be described as:

$S_{\text {inc }}(Q, \omega)=\operatorname{DW}\left[A_{0}(Q) \delta(\omega)+\left(1-A_{0}(Q)\right) L(Q, \omega)\right]$

The Debye-Waller factor $\mathrm{DW}=\exp \left(-\frac{Q^{2}\left\langle u^{2}\right\rangle}{3}\right)$ accounts for the spatial extension of the vibrational modes of the system and $\delta$ is the Dirac delta function. The term $A_{0}$ (see Eq. 3 below) is related to the Elastic Incoherent Structure Factor (EISF) which gives access to the geometry of the motions. EISF is defined as:

$\operatorname{EISF}(Q)=\frac{I_{\mathrm{el}}(Q)}{I_{\mathrm{el}}(Q)+I_{\mathrm{qe}}(Q)}$,

where $I_{\mathrm{el}}$ and $I_{\mathrm{qe}}$ represent, respectively, the elastic and quasielastic scattering intensity.

$L(Q, \omega)=\frac{1}{\pi} \times \frac{\Gamma(Q)}{\Gamma(Q)^{2}+\omega^{2}}$

is a single Lorentzian function with the Half Width at Half Maximum (HWHM), $\Gamma$, which gives access to the amplitude of the motions. The Lorentzian function $L$ appears as a broadening of the elastic peak and, so, is called the "quasielastic" signal, which is related to the relaxational dynamics (internal over-damped and/or diffusive motions). At low $Q$ values $(Q \approx 2 \pi / r)$, the $\mathrm{H}$ atoms appear confined inside a sphere of radius $a$, where they diffuse freely with the diffusion coefficient $D_{\text {sph }}$ (Volino and Dianoux 1980): in this case, $\Gamma$ is equal to a constant $\Gamma_{0}$, which depends on $D_{\text {sph }}$ and the radius $a\left(\Gamma_{0}=4.33 D_{\mathrm{sph}} / a^{2}\right)$. At high $Q$ values, above a $Q_{0}$ value, which is inversely proportional to the size of the sphere $\left(Q_{0}=\pi / a\right)$, a classic atomic diffusion according to $\Gamma(Q)=D_{\mathrm{sph}} Q^{2}$ is recovered.

Experimental data always depend on instrumental resolution which limits the time-window of the detected motions. Fast motions contribute to the background signal and slow motions to the elastic scattering signal, which explains why the EISF function does not achieve zero in the experimental range. To take into account this effect, a parameter $p$ is introduced which corresponds to the nonresolved motions in the experimental conditions: these atoms are considered as non-mobile and contribute to the elastic peak. The $(1-p)$ mobile protons matching the time-window of the spectrometer give rise to the quasielastic signal. The EISF can therefore be written as:

$\operatorname{EISF}(Q)=p+(1-p) A_{0}(Q)$

In the model of diffusion inside a sphere, $A_{0}$ can be described according to the following equation (Volino and Dianoux 1980):

$A_{0}(Q)=\left(\frac{3 j_{1}(Q a)}{Q a}\right)^{2}$

where $j_{1}(x)=(x \cos (x)-\sin (x)) / x^{2}$ is the first order spherical Bessel function.

In the elastic limit $(\omega=0)$ and for $Q \rightarrow 0$, the dynamical structure factor (Eq. 1) can be expressed within the framework of a Gaussian approximation (Smith 1991):

$\lim _{Q \rightarrow 0} S_{\text {inc }}(Q, \omega=0)=\exp \left(-\frac{Q^{2}\left\langle u^{2}\right\rangle}{3}\right)$

where $\left\langle u^{2}\right\rangle$ is the MSDs of overall atoms, mainly hydrogen ones.

\section{Results}

Effect of trehalose/water environment on amplitude and geometry of C-PC motions

In Fig. 1, the incoherent dynamical structure factor $S_{\text {inc }}$ is reported at $T=280 \mathrm{~K}$ for sample \#3, containing the same ratio of trehalose and $\mathrm{D}_{2} \mathrm{O}$, at different $Q$ values (1.59, 2.75, and $3.76 \AA^{-1}$ ). The scattered signal can be described, according to Eq. 1, by the sum of an elastic contribution and a quasielastic Lorentzian contribution, from which we calculated the function $\Gamma$ (Fig. 2) and the EISF (Fig. 3). Whatever the $Q$ value, the signal was mainly elastic with a relatively small quasielastic contribution, which induced poor statistics of the quasielastic spectra even after 3 days of measurements. The signal evolution as a function of $Q$ for other sample compositions (\#1, 2, and 4) and temperatures $(T=311 \mathrm{~K})$ was similar (not shown).

Figure 2 shows the HWHM $\Gamma$ of the Lorentzian term $L$ in Eq. 1 as a function of $Q^{2}$. The data obtained previously for $\mathrm{D}_{2} \mathrm{O}$-hydrated C-PC without trehalose (sample \#1), from another set of experiments on IN13 spectrometer (Dellerue 2000), have been added for comparison. Due to poor statistics, mainly a qualitative analysis is presented. For the sample without trehalose (\#1), $\Gamma$ was almost constant at small $Q$ value (below $Q^{2} \sim 7.5 \AA^{-2}$ ), then increased with $Q^{2}$ at higher $Q$ value $\left(7.5 \AA^{-2}<Q^{2}<16 \AA^{-2}\right)$, which is the 
Fig. 1 Incoherent dynamical structure factor $S_{\text {inc }}(Q, \omega)$ of protonated $\mathrm{C}$-phycocyanin (C-PC) embedded by a matrix of deuterated trehalose and $\mathrm{D}_{2} \mathrm{O}$ (\#3 C-PC/trehalose/ $\mathrm{D}_{2} \mathrm{O}$ 1:0.75:0.75) at different $Q$ values indicated in the panels and at $T=280 \mathrm{~K}$, together with the elastic (dashed line) and quasielastic Lorentzian components (dotted line), and the flat background (dash-dotted line). a Data from -0.1 to $+0.1 \mathrm{meV}$ and $\mathbf{b}$ same data zoomed in over a smaller energy transfer range. Experiments have been performed on IN13 neutron spectrometer (ILL, France) with a resolution of $\sim 8 \mu \mathrm{eV}$

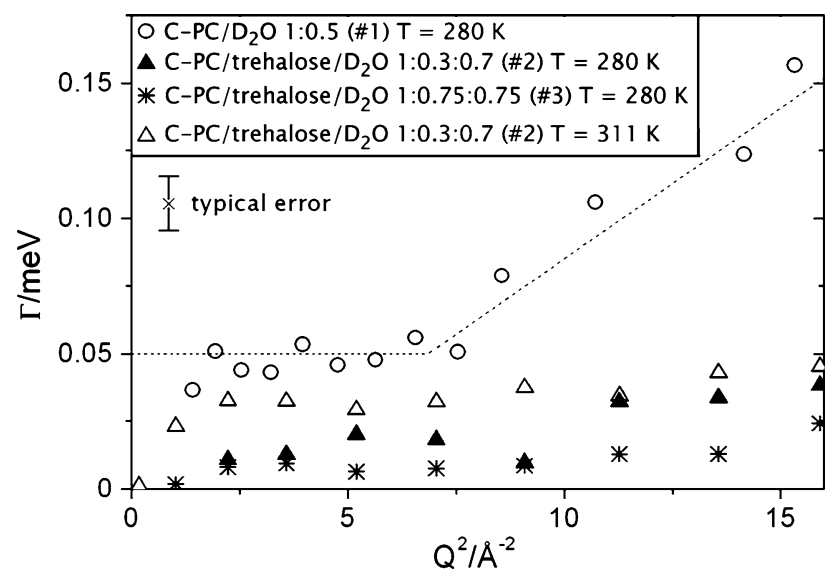

Fig. 2 Half Width at Half Maximum (HWHM) $\Gamma$ of the Lorentzian function describing the broadened component of the scattering signal, as a function of the square modulus of the scattering vector $Q^{2}$ at $T=280$ and $311 \mathrm{~K}$. The dashed line is a guide for the eye for sample \#1 (C-PC/ $\mathrm{D}_{2} \mathrm{O}$ 1:0.5) (sample \#1: data from Dellerue 2000)
C-PC/trehalose/ $\mathrm{D}_{2} \mathrm{O}$ 1:0.75:0.75 (\#3)
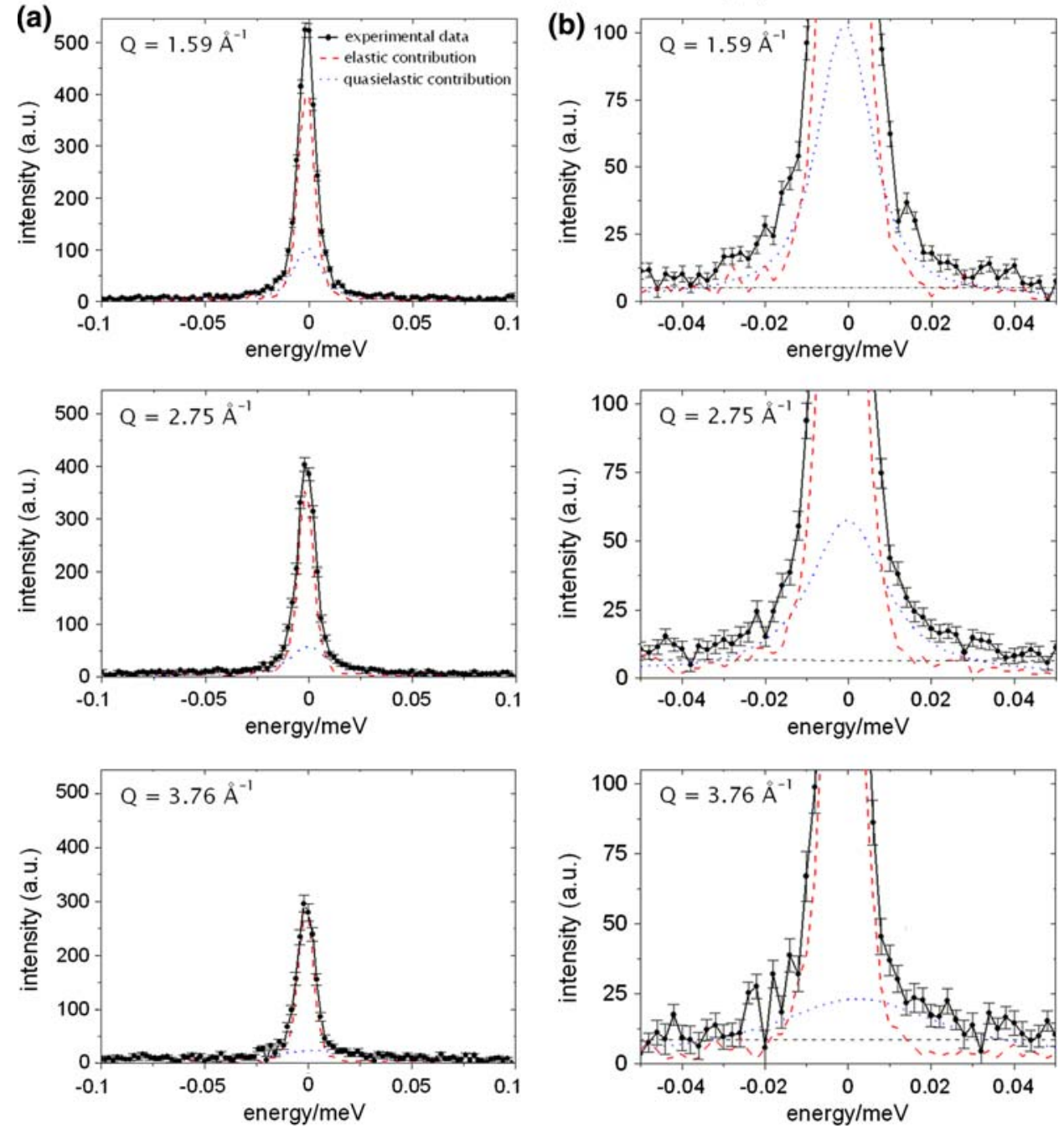

typical behaviour of diffusion inside a sphere (Volino and Dianoux 1980). However, this increase did not follow a $D_{\text {sph }} Q^{2}$ law representative of diffusive motions. Despite a very slight increase above $Q^{2} \sim 10 \AA^{-2}$ for sample \#2, such a transition observed for sample \#1 seemed to be inhibited by trehalose (samples \#2 and 3), even at higher temperature (sample \#2 at $T=311 \mathrm{~K}$ ). However, it was difficult to conclude for sample \#3 since the data were at the limit of the spectrometer resolution (half resolution $\sim 4 \mu \mathrm{eV}$ ). In the low $Q$-region, the $\Gamma_{0}$ value was lower for the trehalose-containing samples (\#2 and 3) than for the sample without trehalose (\#1) (0.01-0.03 and $\sim 0.05 \mathrm{meV}$, respectively), which means that hydrogen motions were slowed down by the sugar. Indeed, the diffusion coefficient $D_{\mathrm{sph}}$ has been evaluated, at $T=280 \mathrm{~K}$, from the $\Gamma_{0}$ value and the sphere radius $a$ (Table 2), with the relation $\Gamma_{0}=4.33 D_{\mathrm{sph}} / a^{2}$. We found $\sim 2 \times 10^{-6} \mathrm{~cm}^{2} \mathrm{~s}^{-1}$ for sample \#1, $\sim 1 \times 10^{-6} \mathrm{~cm}^{2} \mathrm{~s}^{-1}$ for sample \#2, and $\sim 0.6 \times 10^{-6} \mathrm{~cm}^{2} \mathrm{~s}^{-1}$ for sample \#3 (Table 2). We measured also $\Gamma$ for sample \#2 at a higher 
temperature $(T=311 \mathrm{~K})$. As expected, a slightly increased $\Gamma$ value was observed at $T=311 \mathrm{~K}$ than at $T=280 \mathrm{~K}$ (Fig. 2).

Figure 3 represents the EISF from different samples (Fig. 3a) and temperatures (Fig. 3b). The EISF obtained previously for sample \#1 has also been added for comparison (Dellerue 2000). The data were fitted with Eqs. 2 and 3 from the model of diffusion inside a sphere (Volino and Dianoux 1980), as shown for sample \#2. For a better clearness, only the plateau of the curve fitting at large $Q$ value is shown for the other samples, which corresponds to the proportion $p$ of immobile protons. Results from the curve fitting are summarized in Table 2. Due to poor statistics of the quasielastic data, the differences found between the confining sphere radii (Table 2) were not considered to be significant. At $T=280 \mathrm{~K}$, a higher level
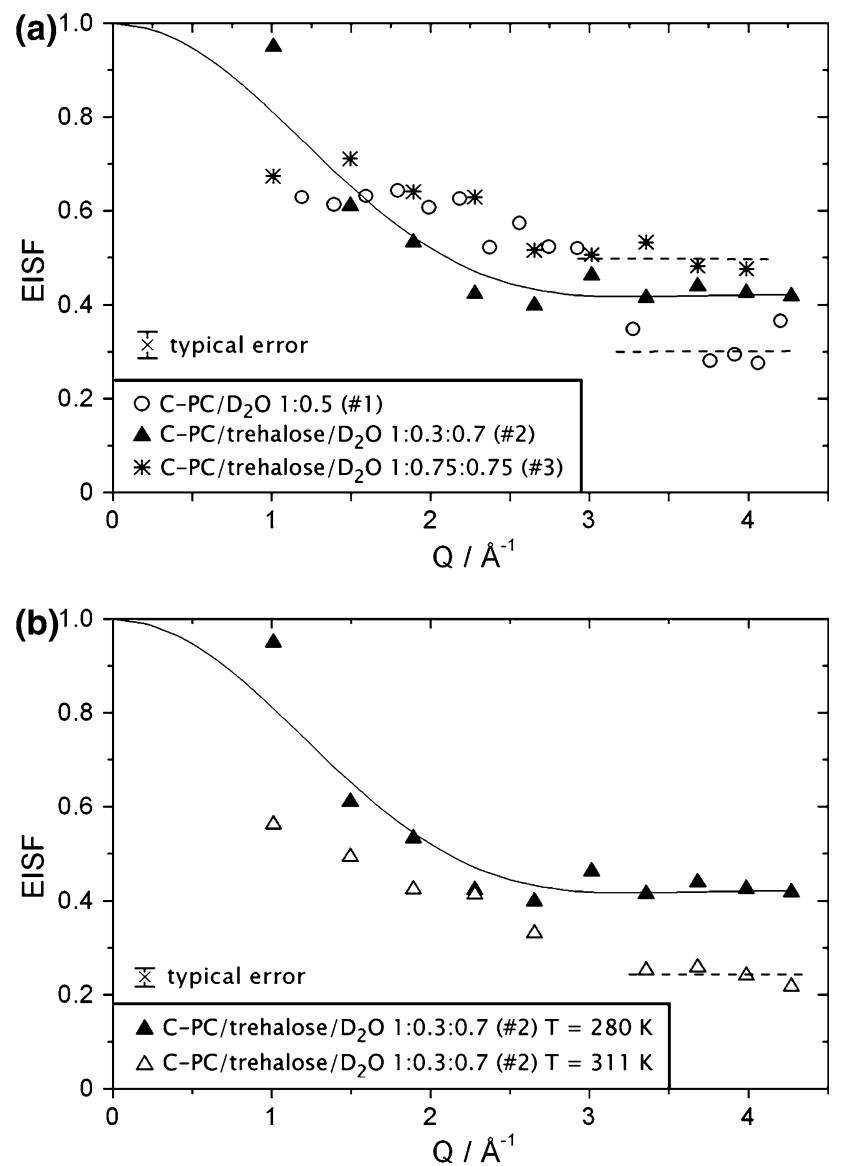

Fig. 3 Elastic Incoherent Structure Factor (EISF) of a protonated $\mathrm{C}$-'PC protein embedded in different compositions of deuterated trehalose $/ \mathrm{D}_{2} \mathrm{O}$ at the same temperature $(T=280 \mathrm{~K})$ and $\mathbf{b}$ of the same sample (\#2 C-PC/trehalose/ $\mathrm{D}_{2} \mathrm{O}$ 1:0.3:0.7) at $T=280$ and $311 \mathrm{~K}$. The full line is the fit of Eqs. 2 and 3 to the data from sample \#2 at $T=280 \mathrm{~K}$, according to the model of diffusion inside a sphere (Volino and Dianoux 1980). For the other data, only the plateau of the fit at high $Q$ value is shown (dashed line), which corresponds to the proportion $p$ of immobile protons (sample \#1: data from Dellerue 2000) of trehalose content induced an increased fraction of immobile protons: $p$ was $\sim 0.3$ for the sample without trehalose $(\# 1), \sim 0.4$ for $15 \%$ trehalose $(\# 2)$, and $\sim 0.5$ for $30 \%$ trehalose (\#3) (Fig. 3a, Table 2). As expected, for sample \#2, the fraction of mobile protons was increased with temperature (from 280 to $311 \mathrm{~K}$ ), as evidenced by the decrease in the parameter $p$ (the number of immobile protons) from $\sim 0.4$ to $\sim 0.25$ (Fig. 3 b, Table 2 ).

Effect of trehalose/water environment on the evolution of C-PC MSDs as a function of temperature

In Fig. 4, we have plotted the logarithm of the elastic intensity $S(Q, \omega=0)$ versus $Q^{2}$ for a few temperatures and the data have been analysed with Eq. 4 over the whole $Q$ range, except at $T=318 \mathrm{~K}$ for which the analysis was only performed up to $Q=4.5 \AA^{-1}$. The slope of the solid lines corresponds to the MSDs $\left\langle u^{2}\right\rangle$. MSD evolution as a function of temperature was reported in Fig. 5 for hydrated and dry samples of trehalose-coated C-PC (\#2, 3, and 4). The MSDs obtained before for $\mathrm{D}_{2} \mathrm{O}$-hydrated C-PC free of trehalose (sample \#1) (Bellissent-Funel 2004; Dellerue 2000) have been added for comparison. Sample \#2 has been tested only at a few temperatures for comparison with the other samples. At low temperature, from $40 \mathrm{~K}$ up to $\sim 225 \mathrm{~K}$, all samples exhibited a harmonic behaviour, represented by a straight line in Fig. 5, whose amplitude was slightly reduced in trehalose-containing samples (\#2, 3 , and 4), as compared to sample \#1. The "dynamical transition" and its associated temperature have been determined by the deviation $( \pm \sim 5 \%)$ from the straight line at low temperature (Doster et al. 1989). For the hydrated samples (\#1, 2, and 3), we found a "dynamical transition" at $T \approx 225 \pm 5 \mathrm{~K}$. In opposition, in the dry trehalosecoated sample (\#4), such a dynamical transition was not observed and the MSDs of this sample remain harmonic up

Table 2 Parameters $a$ (confining sphere radius), $p$ (immobile proton proportion), and $\mathrm{D}_{\text {sph }}$ (diffusion coefficient)

\begin{tabular}{|c|c|c|c|c|c|}
\hline & $\begin{array}{l}\text { Sample composition } \\
(w: w: w)\end{array}$ & $\begin{array}{l}T \\
(\mathrm{~K})\end{array}$ & $\begin{array}{l}a \\
(\AA)\end{array}$ & $p$ & $\begin{array}{l}D_{\mathrm{sph}} \\
\left(\mathrm{cm}^{2} \mathrm{~s}^{-1}\right)\end{array}$ \\
\hline \#1 & $\mathrm{C}-\mathrm{PC} / \mathrm{D}_{2} \mathrm{O} \quad 1: 0.5$ & 280 & 1.1 & 0.30 & $\sim 2.0 \times 10^{-6}$ \\
\hline \#2 & C-PC/trehalose/ $\mathrm{D}_{2} \mathrm{O}$ 1:0.3:0.7 & 280 & 1.4 & 0.40 & $\sim 1.0 \times 10^{-6}$ \\
\hline \#2 & $\mathrm{C}-\mathrm{PC} /$ trehalose $/ \mathrm{D}_{2} \mathrm{O}$ 1:0.3:0.7 & 311 & 1.7 & 0.25 & $\sim 1.4 \times 10^{-6}$ \\
\hline \#3 & $\begin{array}{l}\text { C-PC/trehalose } / \mathrm{D}_{2} \mathrm{O} \\
1: 0.75: 0.75\end{array}$ & 280 & 1.5 & 0.50 & $\sim 0.6 \times 10^{-6}$ \\
\hline
\end{tabular}

$a$ and $p$ were obtained from the curve fitting from Fig. 3, according to the model of diffusion inside a sphere (Volino and Dianoux 1980) (see Eqs. 2 and 3). $D_{\text {sph }}$ was calculated with the relation $\Gamma_{0}=4.33$ $D_{\mathrm{sph}} / a^{2}$ from the parameter $a$ and the value of $\Gamma_{0}$ found on Fig. 2 (sample \#1: data from Dellerue 2000) 


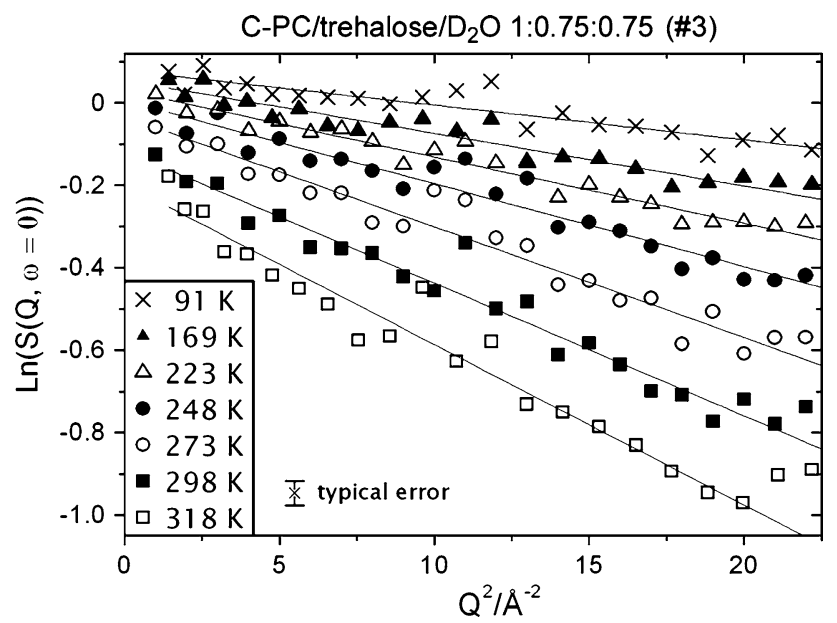

Fig. 4 Analysis of the elastic scattering data, plotted versus the square modulus of the scattering vector $Q^{2}$, at several temperatures, for protonated C-PC embedded by the same ratio of deuterated trehalose and $\mathrm{D}_{2} \mathrm{O}$ (\#3 C-PC/trehalose/ $\mathrm{D}_{2} \mathrm{O}$ 1:0.75:0.75). The slope of the straight lines corresponds to the mean square displacements (MSDs) $\left\langle u^{2}\right\rangle$ in the linear model described by Eq. 4: $\lim _{Q \rightarrow 0} S_{\text {inc }}(Q, \omega=0)=\exp \left(-\frac{Q^{2}\left\langle u^{2}\right\rangle}{3}\right)$. The spectra at $T=223$, 169 , and $91 \mathrm{~K}$ have been scaled vertically for a better visibility by a shift of, respectively, 0.03, 0.06, and 0.09 in the intensity of $\operatorname{Ln}(S(Q, \omega=0)$

to $318 \mathrm{~K}$. The transition temperature was not significantly dependent on the trehalose/water ratio for hydrated samples (\#1, 2, and 3). Furthermore, above the dynamical transition, the MSDs of hydrated samples were not significantly dependent on low content of trehalose, since the behaviour of sample \#2 (15\% trehalose) was close to that of sample \#1. However, the MSDs of the sample \#3 containing a higher ratio of trehalose (30\%) were slightly reduced, compared to the other hydrated samples (\#1 and 2).

\section{Discussion}

In the present study, by using neutron scattering measurements, we measured specifically the motions of the nonexchangeable protons of the C-PC protein embedded in different deuterated trehalose $/ \mathrm{D}_{2} \mathrm{O}$ matrices.

The quasielastic scattering signal, corresponding to a Lorentzian function, reveals a slowing down of internal C-PC dynamics in presence of trehalose. Indeed, the increase of the quasielastic linewidth with $Q^{2}$ observed in sample \#1 (without sugar) is inhibited by trehalose in samples \#2 and 3 (Fig. 2). These data are consistent with another neutron scattering study reporting a constant $\Gamma$ function for lysozyme embedded by deuterated glucose, which is the monomer of the disaccharide trehalose (Marconi et al. 2005). Moreover,

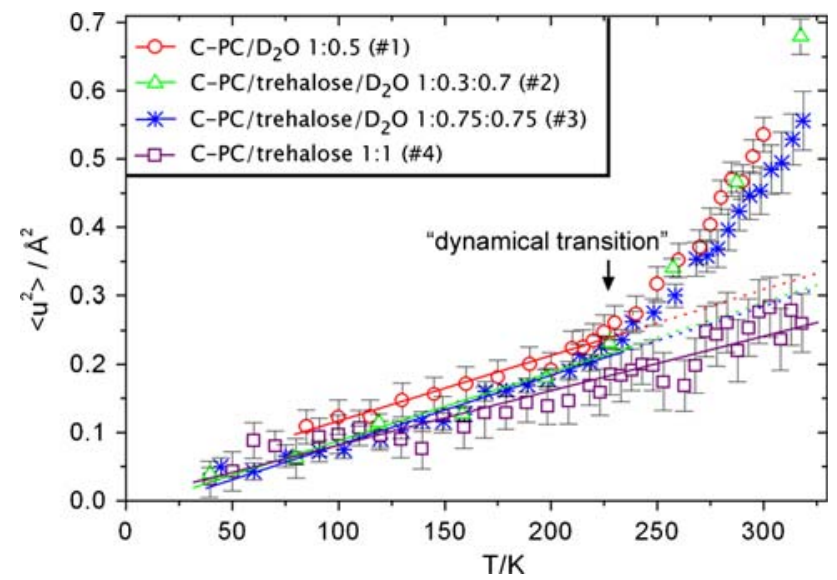

Fig. 5 MSDs $\left\langle u^{2}\right\rangle$ of protonated C-PC samples embedded by deuterated trehalose $/ \mathrm{D}_{2} \mathrm{O}$ at different ratios as a function of temperature $(40-318 \mathrm{~K})$. The solid and dotted lines represent the harmonic behaviour of each sample, respectively, before and after the "dynamical transition" (sample \#1: data from Bellissent-Funel 2004 and Dellerue 2000)

the diffusion coefficient decreases with the amount of trehalose, from $\sim 2 \times 10^{-6} \mathrm{~cm}^{2} \mathrm{~s}^{-1}$ for sample \#1 (without sugar) to $\sim 1 \times 10^{-6} \mathrm{~cm}^{2} \mathrm{~s}^{-1}$ for sample \#2 (15\% trehalose) and $\sim 0.6 \times 10^{-6} \mathrm{~cm}^{2} \mathrm{~s}^{-1}$ for sample \#3 $(30 \%$ trehalose) (Table 2).

In addition, the evolution of the EISF shows a reduced fraction of mobile protons with trehalose, as evidenced by the increase of $p$ (the number of immobile protons) (Fig. 3a, Table 2), which confirms our previous inelastic time-of-flight neutron study (Köper et al. 2002). Yet, we do not probe the same motions, due to the different time ranges: in time-of-flight experiments, we probe all the motions faster than $10 \mathrm{ps}$, whereas we have access to motions from 150 ps up to 400 ps with IN13 spectrometer. As expected, since the $p$ value of immobile protons decreases with the energy resolution, we found $p$ around $0.6-0.8$ in time-of-flight measurements (Köper et al. 2002), whereas, in the present study, $p$ was about $0.3-0.4$ for comparable trehalose/water ratios. Data from the EISF (Fig. 3a, Table 2) do not show significantly different sphere radii between the samples (\#1, 2, and 3), confirming the similar geometry of C-PC motions we found previously by inelastic time-of-flight measurements (Köper et al. 2002). Since a direct interaction with trehalose should induce changes in the geometry of the protein proton motions, our result would mean that the C-PC nonexchangeable $\mathrm{H}$ atoms do not interact directly with the sugar, in opposition to the "water replacement" model (Crowe et al. 1984). However, we did not focus on the motions of the protein exchangeable protons, while, as usually polar, they are likely the sites where the trehalose binds to the protein. 
As measured by elastic neutron scattering, the MSDs of C-PC protein, coated or not with trehalose, exhibit a harmonic behaviour between 40 and $\sim 225 \mathrm{~K}$ (Fig. 5). However, the amplitude is slightly reduced in the samples containing trehalose (\#2, 3, and 4), as compared to the sample without sugar (\#1). This result is in agreement with previous elastic neutron scattering measurements showing lower harmonic motions for both dry $\mathrm{MbCO} /$ trehalose (Cordone et al. 1999) and lysozyme/glycerol (Tsai et al. 2000) than for the corresponding hydrated protein without cryoprotectant. In the dry C-PC/trehalose sample (\#4), the harmonic behaviour of the protein extends at room temperature, without any detectable dynamical transition, as also measured previously for the mixture "trehalose and MbCO" (Cordone et al. 1999). A predominant role of trehalose in this behaviour has been evoked, since motions of dry pure trehalose remain also harmonic up to room temperature (Cordone et al. 1999). Likewise, the MSDs of lysozyme in glycerol follow those of the pure glycerol even above the dynamical transition (Paciaroni et al. 2002), consistently with the study from Caliskan et al. (2004), reporting that proteins are strongly coupled dynamically to trehalose and glycerol. However, the harmonic behaviour of dry protein/ cryoprotectant samples raises the issue of the role of the cryoprotectant versus the role of the absence of water. Therefore, for the present study, elastic neutron scattering measurements on dry C-PC alone, which are presently not available, would allow to compare with sample \#4 (dry C-PC/trehalose).

Indeed, it is well-known that water plays a major role in anharmonic motions and the so-called "dynamical transition". Absence of dynamical transition has been reported in dry proteins, without any bioprotective agent (Ferrand et al. 1993; Gabel et al. 2004). Furthermore, addition of $\mathrm{D}_{2} \mathrm{O}$ to trehalose induces anharmonic motions above a transition temperature (Magazù et al. 2004). It is noteworthy that it is extremely difficult to eliminate all water molecules in a protein sample by lyophilization, since some water molecules are necessary to maintain the structure of the protein. These residual water molecules may be of great importance to modulate the protein dynamics behaviour, as shown by infrared and Raman spectroscopy and flash-photolysis study (Belton and Gil 1994; Librizzi et al. 2002).

The use of light or heavy water in protein samples highly influences the measurement of MSDs by incoherent neutron scattering. Cordone et al. (1999) and Librizzi et al. (2001) described, respectively, the motions of either all $\mathrm{H}$ atoms or the non-exchangeable ones of both $\mathrm{MbCO}$ and trehalose. For the dry MbCO/trehalose sample, Cordone et al. (1999) reported smaller MSDs than Librizzi et al. (2001). That would mean that motions of exchangeable $\mathrm{H}$ atoms, which are likely involved in trehalose-protein interactions, may be slower compared to the non-exchangeable ones. In our study, as we used deuterated trehalose and made "H-D" exchange before sample lyophilization, we probed specifically the non-exchangeable proton motions of the protein only. Therefore, from our study, we may conclude that the MSDs of the non-exchangeable protein $\mathrm{H}$ atoms in the dry $\mathrm{C}$-PC/trehalose sample (\#4) follow a harmonic behaviour like dry trehalose, as measured by Cordone et al. (1999).

Above a transition temperature of $\sim 225 \mathrm{~K}$, the presence of water is crucial, in our study, to observe the large amplitude anharmonic motions of C-PC protein in samples $\# 1$, 2, and 3. These motions may play a key-role by increasing protein flexibility to achieve functional configurations (Doster et al. 1989; Ferrand et al. 1993) but may also be responsible for unfolding and denaturation (LópezDíez et al. 2004). The transition temperature is however not significantly dependent on trehalose $/ \mathrm{D}_{2} \mathrm{O}$ ratio in all hydrated samples (\#1, 2, and 3). The anharmonicity of MSDs is not significantly different between samples \#1 (without trehalose) and \#2 (15\% trehalose), whereas a slight decrease has been observed with $30 \%$ trehalose (sample \#3). The hydration degree of these samples is close to $30 \%$ but differs from a few percents (Table 1), which might influence the amplitude of MSDs, especially at high temperature $(T>270 \mathrm{~K})$. Indeed, it seems as if the higher the hydration level, the higher the MSDs: sample \#2 > sample \#1 > sample \#3 for a hydration level of, respectively, 35, 33, and 30\%. Such an effect has previously been reported, especially for high hydration levels (57-93\%) of the purple membrane (Lehnert et al. 1998). However, this slight decrease of MSDs in sample \#3 seems to be in agreement with the decrease of MbCO MSDs in trehalose/water matrix containing a high amount of trehalose, obtained by molecular dynamics simulations (Cottone et al. 2001). This progressive decrease of MSD amplitude of C-PC suggests a modulation of protein internal motions by trehalose. In hydration conditions, a similar inhibition effect has been observed with glucose and to a lesser extent with glycerol, when present in the matrix of lysozyme (Paciaroni et al. 2006).

How can such an environment modulate protein dynamics? Recently, the "plasticizing character" of the surrounding matrix (Paciaroni et al. 2006), as well as its viscosity (Cornicchi et al. 2005; Rector et al. 2001), have been reported to play a major role in protein dynamics. At the molecular level, the modulating "strength" of the constraints imposed by the sugar/water matrix on the protein has been attributed to some water molecules shared between the protein and the sugar (Cottone et al. 2002; López-Díez et al. 2004). Indeed, the interaction between sugar and water molecules may be stronger than between the water molecules themselves, allowing the sugar to form with water a glassy shell (Affouard et al. 
2005; Branca et al. 1999; Giuffrida et al. 2006; Magazù et al. 2007). This "kosmotrope" character of trehalose, in acquiring many of the available water hydrogen bonds normally directed towards protein surface, might explain the decrease of the non-exchangeable atom motions in sample \#3, as well as the absence of anharmonic behaviour of the motions in sample \#4. Likewise, a kosmotrope action of the phosphate ions has been reported to highly reduce the MSDs of butyrylcholinesterase in presence of water (Gabel et al. 2004).

In conclusion, in the present study, we measured specifically the non-exchangeable $\mathrm{H}$ atom motions of the C-PC protein embedded in different water/trehalose deuterated environments. We found that trehalose decreases $\mathrm{C}$ PC internal dynamics, as shown by a reduced diffusion coefficient $D_{\mathrm{sph}}$ of protein $\mathrm{H}$ atoms. Moreover, the sugar inhibits the increased linewidth $\Gamma$ with $Q^{2}$ observed in the sample without trehalose. The presence of sugar induces a higher proportion of immobile protons in the protein. Furthermore, elastic neutron scattering measurements as a function of temperature show a tiny decrease of MSDs with $30 \%$ trehalose in hydration conditions. Finally, we report a harmonic behaviour for the dry trehalose/C-PC sample up to room temperature, without the presence of a "dynamical transition". These results suggest that a role of trehalose would be to stabilize proteins by inhibiting some fluctuations at the origin of protein unfolding and denaturation (Bieganski et al. 1998; Green and Angell 1989; López-Díez et al. 2004; Sola-Penna et al. 1998).

Acknowledgments We are very grateful to Dr. Henry L. Crespi (Argonne National Laboratory, USA) for providing us with protonated C-PC protein.

\section{References}

Affouard F, Bordat P, Descamps M, Lerbret A, Magazù S, Migliardo F, Ramirez-Cuesta AJ, Telling MFT (2005) A combined neutron scattering and simulation study on bioprotectant systems. Chem Phys 317:258-266

Bellissent-Funel MC, Zanotti JM, Chen SH (1996) Slow dynamics of water molecules on the surface of a globular protein. Faraday Discuss 103:281-294

Bellissent-Funel MC (2004) Internal motions in proteins: a combined neutron scattering and molecular modelling approach. Pramana-J Phys 63:91-97

Belton PS, Gil AM (1994) IR and Raman-spectroscopic studies of the interaction of trehalose with hen egg-white lysozyme. Biopolymers 34:957-961

Bieganski RM, Fowler A, Morgan JR, Toner M (1998) Stabilization of active recombinant retroviruses in an amorphous dry state with trehalose. Biotechnol Prog 14:615-620

Branca C, Magazù S, Maisano G, Migliardo P (1999) Anomalous cryoprotective effectiveness of trehalose: Raman scattering evidences. J Chem Phys 111:281-287

Caliskan G, Mechtani D, Roh JH, Kisliuk A, Sokolov AP, Azzam S, Cicerone MT, Lin-Gibson S, Peral I (2004) Protein and solvent dynamics: How strongly are they coupled? J Chem Phys 121:1978-1983

Carpenter JF, Crowe JH (1989) An infrared spectroscopic study of the interactions of carbohydrates with dried proteins. Biochemistry 28:3916-3922

Clegg JS, Filosa MF (1961) Trehalose in cellular slime mould dictyostelium-mucoroides. Nature 192:1077-1078

Cordone L, Galajda P, Vitrano E, Gassmann A, Ostermann A, Parak F (1998) A reduction of protein specific motions in co-ligated myoglobin embedded in a trehalose glass. Eur Biophys $\mathrm{J}$ 27:173-176

Cordone L, Ferrand M, Vitrano E, Zaccai G (1999) Harmonic behavior in trehalose coated carbon-monoxy-myoglobin at high temperature. Biophys J 76:1043-1047

Cordone L, Cottone G, Giuffrida S, Palazzo G, Venturoli G, Viappiani C (2005) Internal dynamics and protein-matrix coupling in trehalose-coated proteins. Biochim Biophys Acta 1749:252-281

Cornicchi E, Onori G, Paciaroni A (2005) Picosecond-time-scale fluctuations of proteins in glassy matrices: the role of viscosity. Phys Rev Lett 95:158104 1-4

Cornicchi E, Marconi M, Onori G, Paciaroni A (2006) Controlling the protein dynamical transition with sugar-based bioprotectant matrices: a neutron scattering study. Biophys J 91:289-297

Cottone G, Cordone L, Ciccotti G (2001) Molecular dynamics simulation of carboxy-myoglobin embedded in a trehalose-water matrix. Biophys J 80:931-938

Cottone G, Ciccotti G, Cordone L (2002) Protein-trehalose-water structures in trehalose coated carboxy-myoglobin. J Chem Phys 117:9862-9866

Crowe JH, Crowe LM, Chapman D (1984) Preservation of membranes in anhydrobiotic organisms - the role of trehalose. Science 223:701-703

Crowe LM, Reid DS, Crowe JH (1996) Is trehalose special for preserving dry biomaterials? Biophys J 71:2087-2093

Dellerue S (2000) Structure et dynamique de protéines photosynthétiques étudiées par diffusion de neutrons et simulation par dynamique moléculaire. Ph.D. thesis, Université Paris-Sud, Orsay, France

Doster W, Cusack S, Petry W (1989) Dynamical transition of myoglobin revealed by inelastic neutron scattering. Nature 337:754-756

Eroglu A, Russo MJ, Bieganski R, Fowler A, Cheley S, Bayley H, Toner M (2000) Intracellular trehalose improves the survival of cryopreserved mammalian cells. Nat Biotechnol 18:163-167

Ferrand M, Dianoux AJ, Petry W, Zaccai G (1993) Thermal motions and function of bacteriorhodopsin in purple membranes: effects of temperature and hydration studied by neutron scattering. Proc Natl Acad Sci USA 90:9668-9672

Fox KC (1995) Biopreservation. Putting proteins under glass. Science 267:1922-1923

Gabel F, Weik M, Doctor BP, Saxena A, Fournier D, Brochier L, Renault F, Masson P, Silman I, Zaccai G (2004) The influence of solvent composition on global dynamics of human butyrylcholinesterase powders: a neutron-scattering study. Biophys $\mathrm{J}$ 86:3152-3165

Giuffrida S, Cottone G, Librizzi F, L Cordone (2003) Coupling between the thermal evolution of the heme pocket and the external matrix structure in trehalose coated carboxymyoglobin. J Phys Chem B 107:13211-13217

Giuffrida S, Cottone G, Cordone L (2006) Role of solvent on proteinmatrix coupling in $\mathrm{MbCO}$ embedded in water-saccharide systems: a Fourier transform infrared spectroscopy study. Biophys J 91:968-980

Glazer AN (1989) Light guides. Directional energy transfer in a photosynthetic antenna. J Biol Chem 264:1-4 
Gottfried DS, Peterson ES, Sheikh AG, Wang J, Yang M, Friedman JM (1996) Evidence for damped hemoglobin dynamics in a room temperature trehalose glass. J Phys Chem 100:12034-12042

Green JL, Angell CA (1989) Phase-relations and vitrification in saccharide-water solutions and the trehalose anomaly. J Phys Chem 93:2880-2882

Hagen SJ, Hofrichter J, Eaton WA (1995) Protein reaction-kinetics in a room-temperature glass. Science 269:959-962

Hattori A, Crespi HL, Katz JJ (1965) Effect of side-chain deuteration on protein stability. Biochemistry 4:1213-1225

Köper I, Bellissent-Funel MC (2002) Hindered protein dynamics in the presence of a cryoprotecting agent. Appl Phys A 74:S1257S1259

Köper I, Bellissent-Funel MC, Petry W (2005) Dynamics from picoseconds to nanoseconds of trehalose in aqueous solutions as seen by quasielastic neutron scattering. J Chem Phys 122:014514 1-6

Lehnert U, Réat V, Weik M, Zaccai G, Pfister C (1998) Thermal motions in bacteriorhodopsin at different hydration levels studied by neutron scattering: correlation with kinetics and light-induced conformational changes. Biophys J 75:1945-1952

Librizzi F, Paciaroni A, Pfister C, Vitrano E, Zaccai G, Cordone L (2001) Dynamical properties of carbonmonoxy-myoglobin embedded in trehalose matrices of different water content studied by FTIR and elastic neutron scattering. In: Dianoux AJ (ed) Proceedings of the ILL millennium symposium and European user meeting. ILL, Grenoble, pp 58-59

Librizzi F, Viappiani C, Abbruzzetti S, Cordone L (2002) Residual water modulates the dynamics of the protein and of the external matrix in "trehalose coated" MbCO: an infrared and flashphotolysis study. J Chem Phys 116:1193-1200

López-Díez EC, Bone S (2004) The interaction of trypsin with trehalose: an investigation of protein preservation mechanisms. Biochim Biophys Acta 1673:139-148

Magazù S, Maisano G, Migliardo F, Mondelli C, Romeo G (2004) An elastic neutron scattering on dynamical transition in hydrogenbonded systems. J Mol Struct 700:225-227

Magazù S, Migliardo F, Telling MT (2007) Study of the dynamical properties of water in disaccharide solutions. Eur Biophys $\mathrm{J}$ $36: 163-171$
Marconi M, De Francesco A, Cornicchi E, Onori G, Paciaroni A (2005) Hydration and temperature dependent dynamics of lysozyme in glucose-water matrices. A neutron scattering study. Chem Phys 317:274-281

Mazzobre MF, Buera MD, Chirife J (1997) Glass transition and thermal stability of lactase in low-moisture amorphous polymeric matrices. Biotechnol Prog 13:195-199

Paciaroni A, Cinelli S, Onori G (2002) Effect of the environment on the protein dynamical transition: a neutron scattering study. Biophys J 83:1157-1164

Paciaroni A, Cornicchi E, De Francesco A, Marconi M, Onori G (2006) Conditioning action of the environment on the protein dynamics studied through elastic neutron scattering. Eur Biophys J 35:591-599

Pérez J, Zanotti JM, Durand D (1999) Evolution of the internal dynamics of two globular proteins from dry powder to solution. Biophys J 77:454-469

Rector KD, Jiang JW, Berg MA, MD Fayer (2001) Effects of solvent viscosity on protein dynamics: infrared vibrational echo experiments and theory. J Phys Chem B 105:1081-1092

Sola-Penna M, Meyer-Fernandes JR (1998) Stabilization against thermal inactivation promoted by sugars on enzyme structure and function: why is trehalose more effective than other sugars? Arch Biochem Biophys 360:10-14

Smith JC (1991) Protein dynamics - comparison of simulations with inelastic neutron scattering experiments. Q Rev Biophys 24:227291

Tsai AM, Neumann DA, Bell LN (2000) Molecular dynamics of solid-state lysozyme as affected by glycerol and water: a neutron scattering study. Biophys J 79:2728-2732

Volino F, Dianoux AJ (1980) Neutron incoherent scattering law for diffusion in a potential of spherical symmetry: general formalism and application to diffusion inside a sphere. Mol Phys 41:271279

Zaccai G (2000) How soft is a protein? A protein dynamics force constant measured by neutron scattering. Science 288:16041607 